\title{
Fomento de la concienciación lingüística del profesorado en formación. Estudio de caso*
}

ZÓSIMO LÓPEZ PEÑA*

Recepción: 26 de octubre de 2018

Aprobación: 7 de marzo de 2019

Forma de citar este artículo: López, Z. (2019). Fomento de la concienciación lingüística del profesorado en formación. Estudio de caso. Cuadernos de Lingüística Hispánica, (34), pp 155-174.

(하 $10.19053 / 0121053 X . n 34.2019 .8542$

* Artículo de reflexión.

** El autor es doctor en Comunicación e Industrias Creativas por la Universidad de Santiago de Compostela y profesor en el Departamento de Didáctica de la Lengua y la Literatura de la Universidad Internacional de La Rioja (UNIR) (España). Sus líneas de investigación son la didáctica de la publicidad y la implantación y mejora del Plan Lingüístico de Centro. Correo electrónico: zosimo.lopez@unir.net. https://orcid.org/0000-0001-6058-9003 


\section{Resumen}

Si bien son varias las publicaciones que se han ocupado de estudiar la importancia del Plan Lingüístico de Centro (PLC) como instrumento para fomentar la Competencia en Comunicación Lingüística, no son tantas las investigaciones que tratan de los beneficios de esta herramienta para trabajar la concienciación lingüística, a pesar de ser esta una cuestión fundamental en la formación de los docentes.

El objetivo del presente artículo consiste en analizar el modo en que hemos podido trabajar la concienciación lingüística a través de la propuesta y puesta en práctica de los distintos elementos que integran los materiales didácticos de la asignatura Diseño y Elaboración de un plan Lingüístico de Centro y, muy particularmente, mediante el análisis de las ciento dieciocho actividades realizadas por cuarenta y cinco alumnos de esta asignatura en el periodo aproximado de cuatro meses. En primer lugar proponemos un marco teórico en el que tratamos los conceptos de conciencia y concienciación lingüística, cómo trabajar esta última mediante el PLC y de qué forma esta se refleja en los currículum de Infantil y Primaria. Para evaluar nuestro objetivo presentaremos un esbozo de la estructura de nuestros materiales en cuanto a contenidos y actividades y los resultados del análisis de una de las actividades realizadas por los alumnos; aquella que consideramos más significativa para demostrar que hemos fomentado la concienciación lingüística de nuestro alumnado.

Palabras clave: comportamiento lingüístico, lenguas indígenas, educación a distancia, educación intercultural, multiculturalismo.

\section{Promoting the Linguistic Awareness of Teachers in Training. Case Study}

\section{Abstract}

Although there are several publications that have studied the importance of the Center's Linguistic Plan (PLC, by its Spanish acronym) as an instrument to promote the Competence in Linguistic Communication, there are not many researches that deal with the benefits of this tool to work on Linguistic awareness, although this is a fundamental issue in teacher's training.

The objective of this article is to analyze the way we have been able to work on linguistic awareness through the proposal and putting into practice of the different elements that make up the didactic materials of the subject called Design and Development of a Center's Linguistic Plan and, very particularly, through the analysis of the one hundred 
and eighteen activities carried out by forty five students of this subject in the approximate period of four months.

First of all, we propose a theoretical framework in which we deal with the concepts of conscience and linguistic awareness, how to work this last one through the PLC and how it is reflected in the Infant and Primary curricula. To evaluate our objective we will present an outline of the structure of our materials in terms of contents and activities and the results of the analysis of one of the activities carried out by the students; the one we consider most significant to demonstrate that we have fostered the linguistic awareness of our students.

Keywords: linguistic behavior, indigenous languages, distance education, intercultural education, multiculturalism.

\section{Promotion de la prise de conscience linguistique du corps enseignant en formation. Étude de cas}

\section{Résumé}

Bien qu'il y ait de diverses publications qui se sont occupées d'étudier l'importance du Plan Linguistique de Centre (PLC) en tant qu'instrument pour promouvoir la Compétence en Communication Linguistique, il n'y a pas autant de recherches qui abordent les bénéfices de cet outil pour travailler la prise de conscience linguistique, malgré le fait que ceci soit une question fondamentale dans la formation des enseignants.

L'objectif de cet article consiste à analyser la façon dont nous avons pu travailler la prise de conscience linguistique à travers la proposition et la mise en pratique des divers éléments qui intègrent les matériaux didactiques du cours Design et Élaboration d'un plan Linguistique de Centre et, plus particulièrement, au moyen de l'analyse de cent dixhuit activités réalisées par quarante-cinq apprenants de ce cours-là pendant à peu près quatre mois. D'abord nous proposons un cadre théorique dans lequel nous abordons les concepts de conscience et prise de conscience linguistique, comment travailler celle-ci au moyen du PLC, et de quelle manière elle se reflète dans les curriculums d'Infantile et Primaire. Pour évaluer notre objectif on présentera une esquisse de la structure de nos matériaux quant aux contenus et activités et les résultats de l'analyse d'une des activités réalisées par les apprenants ; celle que nous considérons la plus significative pour démontrer que nous avons promu la prise de conscience linguistique de nos étudiants.

Mots clés: comportement linguistique, langues autochtones, éducation à distance, éducation interculturel, multiculturalisme. 


\section{Promoção da consciência linguística dos professores em treinamento. Estudo de caso}

\section{Resumo}

Embora existam várias publicações preocupadas em estudar a importância do Plano Linguístico do Centro (PLC) como um instrumento para promover a Competência em Comunicação Linguística, não existem muitas investigações que tratem dos benefícios dessa ferramenta para trabalhar na conscientização da linguagem. apesar de ser uma questão fundamental na formação de professores. 0 objetivo deste artigo é analisar a maneira pela qual conseguimos trabalhar na conscientização da linguagem por meio da proposta e implementação dos diferentes elementos que integram os materiais de ensino da disciplina Design e desenvolvimento de um plano de linguagem central e, muito particularmente, analisando as cento e dezoito atividades realizadas por quarenta e cinco estudantes desta matéria no período aproximado de quatro meses. Primeiro, propomos um arcabouço teórico no qual lidamos com os conceitos de conscientização e consciência linguística, como trabalhar esse último através do PLC e como ele se reflete nos currículos de ensino básico. Para avaliar nosso objetivo, apresentaremos um esboço da estrutura de nossos materiais em termos de conteúdo e atividades e os resultados da análise de uma das atividades realizadas pelos alunos; aquele que consideramos mais significativo para mostrar que promovemos a consciência linguística de nossos alunos.

Palavras-chave: comportamento linguístico, línguas indígenas, educação a distância, educação intercultural, multiculturalismo. 


\section{Introducción}

El Proyecto Lingüístico de Centro (PLC) es un documento-herramienta en el que se plasma todo el conjunto de acciones destinadas a mejorar tanto la competencia en comunicación lingüística (CCL) como la concienciación lingüística y el comportamiento lingüístico dentro de un centro escolar. Ambas cuestiones constituyen dos de los principales desafíos a los que se enfrentan los docentes de Educación Infantil y Primaria. Por tal motivo es importante poder ofrecer a los presentes y futuros docentes de áreas lingüísticas pertenecientes a estas etapas educativas las herramientas necesarias para crear un PLC. Con tal objetivo se planteó la asignatura Diseño y Elaboración de un Plan Lingüístico de Centro que se cursa en el Máster Universitario en Didáctica de la Lengua en Enseñanza Infantil y Primaria y se imparte en la Universidad Internacional de La Rioja (UNIR).

Si bien son varias las publicaciones que se han ocupado de estudiar la importancia del PLC como instrumento para fomentar la CCL, no son tantas las investigaciones que tratan de los beneficios de esta herramienta para trabajar la concienciación lingüística, a pesar de ser esta una cuestión fundamental en la formación de los docentes tanto para que estos sean capaces de enfrentarse a la realidad de su centro como para que puedan tratar en el aula diversos aspectos que se mencionan explícita o implícitamente en los currículum de Educación Infantil y Primaria en España, tal y como veremos más adelante.

El objetivo del presente artículo consiste en analizar el modo en que hemos podido trabajar la concienciación lingüística a través de la propuesta y puesta en práctica de los distintos elementos que integran los materiales didácticos de la asignatura Diseño y Elaboración de un Plan Lingüístico de Centro, no solo mediante los contenidos sino muy particularmente mediante las actividades planteadas. En primer lugar proponemos un marco teórico en el cual tratamos los conceptos de conciencia y concienciación lingüística, cómo trabajar esta última mediante el PLC y de qué forma esta se refleja en los currículum de Infantil y Primaria. Para evaluar nuestro objetivo presentaremos un breve esbozo de la estructura de nuestros materiales en cuanto a contenidos y actividades y los resultados del análisis de una de las actividades realizadas por los alumnos; aquella que consideramos más significativa para demostrar que hemos logrado fomentar la concienciación lingüística de nuestro alumnado. 


\section{Marco teórico}

\subsection{Sobre los conceptos de conciencia lingüística y concienciación Lin- guística}

Una primera definición de conciencia lingüística es la que nos ofrece Crystal, cuya significativa aportación tiene una relevante importancia didáctica. Así pues, el lingüista se refiere al término como la contestación que, de forma juiciosa, hacemos sobre el uso de la lengua en distintos sentidos, así como al conocimiento de la terminología lingüística.

Término usado sobre lodo en la lingüística educativa para hacer referencia a una respuesta informada, sensata y crítica al uso de la lengua por uno mismo y por los demás, incluyendo el conocimiento de la terminología relevante (conciencia metalingüística). En los primeros años noventa, se dio un especial impulso a la tarea de impulsar la conciencia lingüística justo cuando se empezaron a adoptar nuevas ideas sobre la enseñanza de lenguas en las escuelas. (Crystal, 2000, p. 126)

Este concepto proviene de una tradición anglosajona, concretamente del vocablo "language awareness" y, como veremos un poco más adelante, presenta ciertos matices que han afectado su traducción al español y que deben considerarse. La Association for Language Awareness define la conciencia lingüística (language awareness) como el explícito conocimiento de una lengua, la percepción y sensibilidad conscientes al aprender una lengua, al enseñar una lengua y al utilizar una lengua (Association for Language Awareness, s.f.). Esta descripción redunda, pues, en la importancia que adquiere el término en el ámbito escolar. Así, la conciencia lingüística permitiría percibir elementos de una lengua que, quizás, en antiguas formas de enseñanza podrían pasar inadvertidos. Esto es, la conciencia lingüística posibilita, en cierta medida, la reflexión sobre el conocimiento de una lengua en su capacidad global, desde el aprendizaje del código meramente lingüístico a sus matices supradiscursivos, comunicativos y culturales. A este respecto, los beneficios que se podrían atribuir al trabajo de la conciencia lingüística a la hora de enseñar una lengua, tanto L1 como L2, se pueden resumir en cinco puntos, cinco dimensiones, que se solapan en algunos aspectos, y son las que a continuación se exponen (Centro Virtual Cervantes, s. f.; Garret, 2006; James \& Garrett, 1991).

En primer lugar debe mencionarse la dimensión performativa, que implica que el conocimiento de una lengua por medio de la conciencia lingüística facilita el uso y aprendizaje de esa lengua. Por otra parte ha de citarse la dimensión cognitiva, que hace referencia a la conciencia de patrones, contrastes, sistemas, unidades, categorías y normas de una lengua, así como al desarrollo de una competencia analítica, que va más allá del 
aprendizaje de una lengua. En tercer lugar aludimos a la dimensión afectiva, la relacionada con las actitudes, la motivación y curiosidad acumuladas por medio de la conciencia lingüística. Por su parte, la dimensión social suele estar en relación con la armonización social en contextos de diversidad lingüística y la construcción de mejores relaciones entre grupos culturales diferentes. Redunda en un amor por la diferencia para que, de esta manera, se pueda implementar una mejora de relaciones entre comunidades fomentando el plurilingüismo, la interculturalidad y la multiculturalidad. Finalmente, la dimensión del poder está enfocada en el incremento de la sensibilidad y el empoderamiento de los hablantes para contrarrestar el uso manipulativo de las lenguas. En este contexto preciso se sitúa la llamada conciencia lingüística crítica ${ }^{1}$, que tiene como objetivo principal el trabajo de esta dimensión.

Como habíamos adelantado anteriormente, dado que el término language awareness presenta diversos significados, este se ha traducido al español en algunas ocasiones como concienciación lingüística. A este respecto, Sánchez apunta lo siguiente:

Muchas, variadas y bastante complejas son las definiciones que se han intentado dar tanto del término language awareness como del language learning awareness y algunos conceptos como los de "concienciación lingüística" o "reflexión metalingüística sobre el proceso de aprendizaje de una lengua" figuran como sinónimos en lengua española. Según Eva Vetter, autora de una de las investigaciones más recientes sobre el concepto, la concienciación lingüística [...] resultaría de una zona compartida por el plurilingüismo, la language awareness (entendida ésta como un término compuesto por la afección, la cognición y la actuación el cual es activo en un área lingüístico-cultural) y la actitud frente a la lengua en proceso de aprendizaje. (Sánchez, 2010, pp. 91-92)

Si adoptamos una perspectiva vinculada con la antropología o la etnografía lingüística, podríamos definir el término como la sensibilidad y la concienciación que tiene una persona con respecto a la naturaleza de la lengua y su papel en la vida humana (Kumaravadivelu, 1994; Hawkins, 1984; James \& Garret, 1991). Asimismo, se puede afirmar que en la enseñanza de las lenguas basada en la concienciación lingüística se hace énfasis en la comprensión, en los principios generales y en la experiencia operativa (Kumaravadivelu, 1994), por lo que se observa una finalidad altamente funcional relacionada con el enfoque comunicativo propuesto para el trabajo en las asignaturas de áreas lingüísticas en un centro escolar. Si retomamos a Crystal, este autor pone de manifiesto que "es evi-

1 En inglés, critical language awareness, término acuñado por el lingüista Norman Fairclough, que entronca con el análisis crítico del discurso. 
dente que existe una diferencia entre conciencia lingüística y concienciación lingüística" (Crystal, 2000, p. 133) y que, además, la mejor manera de diseminar dicha concienciación lingüística es por medio de las diferentes manifestaciones artísticas, puesto que el arte alcanza a todo tipo de público. El lingüista entiende, pues, la concienciación como la "acción y efecto de concienciar" (RAE, s.f.) y la conciencia en cuanto "conocimiento claro y reflexivo de la realidad" (RAE, s.f.). En este trabajo no nos ocupamos del fomento de la concienciación lingüística por medio de la música, la pintura, el cine o la ópera, como promulga Crystal, pero sí a través de la formación en el diseño y la elaboración de los planes lingüísticos que tienen o pueden llegar a tener los centros escolares. Se pretende, pues, concienciar lingüísticamente a los docentes que siguen nuestro programa de máster a través de la asignatura propuesta, para que, más tarde, ellos elaboren sus PLC y puedan, asimismo, concienciar a sus alumnos en el aula siguiendo las cinco dimensiones antes mencionadas. Así pues interesa fomentar la reflexión en torno al conocimiento y aprendizaje de una lengua, las normas de una lengua, la actitud hacia una lengua, la convivencia entre distintas lenguas como parte de contextos plurilingüísticos y multiculturales y el uso opresivo de las lenguas.

\subsection{El Proyecto Lingüístico de Centro y la concienciación lingüística}

Entre las distintas herramientas de las que dispone un centro escolar para trabajar la concienciación lingüística y la competencia en comunicación lingüística (CCL) se encuentra el PLC; en este punto radica la necesidad de formar a los docentes de Primaria e Infantil en la elaboración de un PLC. Este consiste en un documento-herramienta imbricado en el denominado Proyecto Educativo de Centro (PEC). En él se compila la regulación de los usos lingüísticos comunicativos, así como el tratamiento y didáctica de las lenguas en todos los aspectos de la vida del centro educativo como, por ejemplo, enseñanza, asignaturas, comunicación interna, comunicación externa, documentos, administración 0 actividades. La finalidad última será la de mejorar la actividad docente, así como la competencia comunicativa de los miembros de la comunidad educativa (Jiménez, 2010, p. 6). El PLC comienza a trabajarse en el mundo anglosajón a finales de los años sesenta del siglo pasado, para abordar dificultades relacionadas con el bilingüismo o plurilingüismo tanto en el ámbito social como en el de la educación formal (Trujillo \& Rubio, 2014, p. 30). Por su parte, España empieza a utilizarlo en los años noventa en comunidades autónomas con lengua cooficial (Vez, 2004; Trujillo \& Rubio, 2014, p. 30; Trujillo, 2015), para proteger las lenguas con un menor número de hablantes; pero, poco a poco y, tras observar su utilidad para aulas con una gran densidad multicultural, el uso del PLC se va extendiendo a centros escolares de otras partes del territorio español. 
En este sentido resulta fundamental tener en cuenta las potencialidades que presenta el PLC en el momento de fijar una conciencia lingüística con respecto a su lengua materna, pero también una concienciación lingüística en relación con otras lenguas que pueden aparecer en el centro escolar, bien porque las dicta el currículo, bien porque las circunstancias hacen que en un colegio se encuentren hablantes de diferentes lenguas y culturas. Como apuntan Trujillo y Rubio (2014, p. 32), se pueden destacar tres puntos principales para atender la diversidad desde el PLC. Como parte del Proyecto Educativo de Centro, el PLC es una herramienta fundamental de escuela inclusiva, puesto que las lenguas son un factor fundamental de inclusión. Asimismo responde a una necesidad real de buena parte del alumnado: acabar con sus problemas relacionados con el uso de la lengua, oral o escrita. Por último, la atención a la diversidad necesita una actuación global que conlleve distintos ámbitos de intervención, como se hace con el PLC.

Podemos esgrimir diferentes razones que defienden la necesidad de implantar un PLC en un centro escolar, pero nos vamos a centrar principalmente en cuatro (López \& Trujillo, 2012, p. 11). Dos de ellas pueden ser, por una parte, la propia complejidad que implica el trabajo con la competencia en comunicación lingüística (CCL,) ya que no todos los alumnos egresados de un centro escolar tienen un dominio absoluto de una lengua (aunque sea su lengua materna); por otra parte debe mencionarse el trabajo y actualización de la CCL en diferentes ámbitos situaciones y registros, debido a que hay que incitar a los alumnos a que manejen con efectividad sus códigos comunicativos en contextos diversos. Otras dos de las razones tienen una vertiente vinculada íntimamente con lo que en este trabajo queremos evidenciar, que es la importancia del PLC para trabajar la concienciación lingüística y la educación intercultural. Por una parte, el dominio de las lenguas, siempre parcial y en continua construcción, se actualiza en diferentes contextos lingüísticos y culturales en los cuales el hablante tiene que activar su competencia para garantizar el éxito en la comunicación. Para finalizar, podemos añadir que la CCL, según el Marco Común Europeo de Referencia (MCER), implica una competencia plurilingüe y una competencia pluricultural. Esto puede implicar cierto desequilibrio, debido a que el alumnado, generalmente, consigue un mayor dominio en una lengua que en las demás. Asimismo, el perfil de competencias en una lengua es diferente del de otras. Además, el perfil pluricultural difiere del perfil plurilingüe. Piénsese en las situaciones que se dan cuando se obtiene un buen conocimiento de la cultura de una comunidad, pero un conocimiento pobre de su lengua 0 , al revés, un pobre conocimiento de una comunidad cuya lengua predominante, sin embargo, se domina (MEC, 2002, p. 143). Dicho esto, es inevitable pensar en la trascendencia que puede tener un PLC fuera de las fronteras de un centro educativo y es que este documento-herramienta 
va más allá del ámbito lingüístico y tiene sus raíces en la realidad social del pasado, el presente y el futuro de la población escolar. Requiere la coordinación y la flexibilidad, y explora estrategias de construcción colectiva del conocimiento. Implica el análisis de la realidad sociocultural y político-económica para definir la intervención educativa en ámbitos lingüísticos, comunicativos y digitales. (Lorenzo i Gales, 2015, p. 23)

\subsection{La presencia de la concienciación lingüística en el currículum de Educación Infantil y Primaria}

Tal y como hemos explicado anteriormente, la necesidad del proceso de concienciación lingüística de los docentes de Educación Infantil y Primaria, que podemos llevar a cabo a través de la asignatura Diseño y Elaboración de un Plan Lingüístico de Centro, resulta fundamental debido a la actual realidad lingüística de los centros y aulas de Educación Infantil y Primaria, una circunstancia que podremos enfrentar de forma más efectiva mediante el PLC.

A su vez, estos docentes deben fomentar la conciencia lingüística de sus alumnos tal y como está recogido en los respectivos textos legislativos, un proceso en el que también adquiere una extraordinaria relevancia el PLC, por cuanto a través del él, los docentes han reflexionado de forma crítica previamente acerca de la lengua. En este sentido puede recordarse que el Real Decreto 1630/2006, de 29 de diciembre, por el que se establecen las enseñanzas mínimas del segundo ciclo de Educación Infantil recoge la siguiente afirmación en su área de conocimiento Lenguajes: Comunicación y Representación, en la que queda reflejada la anteriormente mencionada dimensión afectiva: "Asimismo, es necesario el desarrollo de actitudes positivas hacia la propia lengua y la de los demás, despertando sensibilidad y curiosidad por conocer otras lenguas" (Real Decreto 1630/2006, BOE n. ${ }^{\circ}$, de 4 de enero de 2007, p. 480).

Asimismo, entre los objetivos es posible apreciar, además de la afectiva, la performativa, la cognitiva y, al igual que la social. Entre estos se toman los siguientes:

1. Utilizar la lengua como instrumento de comunicación, de representación, aprendizaje y disfrute, de expresión de ideas y sentimientos, y valorar la lengua oral como un medio de relación con los demás y de regulación de la convivencia.

3. Comprender las intenciones y mensajes de otros niños y adultos, adoptando una actitud positiva hacia la lengua, tanto propia como extranjera. 
7. Iniciarse en el uso oral de una lengua extranjera para comunicarse en actividades dentro del aula, y mostrar interés y disfrute al participar en estos intercambios comunicativos. (Real Decreto 1630/2006, BOE n. ${ }^{0} 4$, de 4 de enero de 2007, p. 480)

Por su parte, el Real Decreto 126/2014, de 28 de febrero, por el que se establece el currículo básico de la Educación Primaria, hace particular hincapié en las dimensiones afectiva y social en el bloque 4 (Conocimiento de la lengua), como contenido: "Conocimiento general de realidad plurilingüe de España y su valoración como fuente de enriquecimiento personal y como una muestra de la riqueza de nuestro patrimonio histórico y cultural" (Real Decreto 126/2014, BOE n. ${ }^{0}$ 52, de 1 de marzo de 2014, p. 19384); en cuanto criterio de evaluación: "6. Conocer la variedad lingüística de España y del español como fuente de enriquecimiento cultural. Mostrar respeto tanto hacia las lenguas y dialectos que se hablan en España, como hacia el español de América” (Real Decreto 126/2014, BOE n. ${ }^{\circ}$ 52, de 1 de marzo de 2014, p. 19384) y, finalmente, como estándar de aprendizaje: "6.1. Conoce y valora la variedad lingüística de España y el español de América; 6.2. Reconoce e identifica algunas de las características relevantes (históricas, socio-culturales [sic], geográficas y lingüísticas) de las lenguas oficiales en España" (Real Decreto 126/2014, BOE n. ${ }^{\circ}$ 52, de 1 de marzo de 2014, p. 19384).

\section{Metodología y materiales didácticos para fomentar la con- cienciación lingüística a través de la asignatura Diseño y Elaboración de un Plan Lingüístico de Centro}

\subsection{Contextualización, objetivos, destinatarios y contenidos de la pro- puesta}

La UNIR oferta programas de grado y máster a más de diez mil alumnos internacionales. El modelo de enseñanza virtual que ofrece la universidad está integrado por clases en línea en directo que pueden ser visualizadas posteriormente, foros empleados para la resolución de dudas, lecciones magistrales y un conjunto de recursos complementarios que apoyan el proceso de enseñanza-aprendizaje y permiten partir de lo esencial y llegar después a lo complementario. Este modelo posibilita a nuestros alumnos realizar un recorrido flexible a través de la información de la cual disponen, así como el empleo de estos datos con distintos fines. Asimismo, el sistema de evaluación que se propone es continua e integra las actividades, lo que nos facilita obtener una evaluación formativa. A este ítem añadimos la prueba de un examen final, lo que nos ofrece una evaluación sumativa. 
El objetivo principal de nuestra propuesta didáctica fue la creación de un material docente para la asignatura Diseño y Elaboración de un Plan Lingüístico de Centro que se imparte en el Máster Universitario en Didáctica de la Lengua en Educación Infantil y Primaria que se enseña en la UNIR. Los destinatarios de este material didáctico fueron los 45 alumnos y alumnas que cursaron esta asignatura desde el 27 de marzo de 2017 al 16 de julio del mismo año, 21 de los cuales asistían a las clases virtuales desde Latinoamérica y 24 de ellos hacían lo propio desde España, lugares de los que procedían respectivamente. Estos alumnos tenían una media de edad de 36 años: los alumnos españoles tenían una media de 32 y los alumnos hispanoamericanos, una media de 39 años. El 85 \% eran mujeres frente a un $15 \%$ que eran hombres.

El modelo de enseñanza a distancia exige al docente el conocimiento de la diversa realidad social, lingüística y educativa de los alumnos que integran el grupo, por cuanto esto favorece el proceso de enseñanza-aprendizaje. En el ejemplo que ofrecemos podemos observar dicha diversidad, gracias a los datos mencionados en el párrafo anterior en cuanto al origen del alumnado, una heterogeneidad que no solo es geográfica sino también lingüística. Esta situación nos obligaba a crear una serie de contenidos abiertos y versátiles que favoreciesen la incorporación de todo el grupo y fueran aplicables a realidades sociolingüísticas muy diferentes.

Con este objetivo se crearon las ideas clave correspondientes a los ocho temas, con sus lecciones magistrales y materiales complementarios, denominados: Plan lingüistico de centro: ¿por qué y para qué existe esta berramienta?; Currículum y PLC; Análisis de realidad para diseñar un PLC; Diseño del PLC: agentes y procesos; Recursos, herramientas y actividades para ejecutar el PLC; Implantación del PLC; Buenas prácticas a la bora de diseñar, ejecutar e implantar un PLC; y Evaluación del PLC.

\section{Resultados conseguidos}

La UNIR emplea, como instrumentos para medir la evaluación continua, dos asistencias obligatorias a las clases virtuales en modo sincrónico, ocho pruebas tipo test, cinco trabajos, la participación en un foro obligatorio y una prueba final en forma de examen.

Es importante considerar que el aprendizaje en entornos de enseñanza a distancia supone un proceso de construcción por parte del discente de forma que este alcance un aprendizaje significativo (Zapata, 2016, p. 13). Esta cuestión resulta ampliamente favorecida, por ejemplo, por las actividades (Onrubia, 2016, p. 8). 
En efecto, es posible que unos de los pilares más importantes de la fijación de contenidos de esta materia sean, precisamente, los trabajos propuestos, puesto que todos ellos entrañan tareas auténticas y están planteados de una manera constructivista, de forma que el primer trabajo sirva para comprender el siguiente y que los sucesivos necesiten conocimientos tratados en los anteriores. Es importante considerar que a través de ellos pretendemos fomentar la concienciación lingüística en sus diferentes dimensiones. Estas actividades aportan una información suficiente para conocer los contextos, los retos, las debilidades y las fortalezas a las que se enfrenta este alumnado a la hora de diseñar, elaborar e implantar un PLC dentro de su presente o posible centro educativo, tal y como se reflexiona a continuación. Importa considerar que las actividades están planteadas de manera que, al finalizarlas, el alumno haya recorrido todas las fases para completar un PLC con aplicación real en su contexto. Se establece, por tanto, una secuenciación lógica que comienza por un reconocimiento del uso de las diferentes lenguas en el currículo educativo del territorio donde trabaja el alumno de este máster (o en donde le gustaría trabajar). Se sigue dotando al alumno de los indicadores suficientes para que pueda realizar un análisis de la realidad. A continuación se le proponen diversas eventualidades que pueden ocurrir a lo largo de la implantación del PLC y que el alumnado debe tener previstas para saber solucionarlas con efectividad y, finalmente, se plantea una actividad basada en aprender a evaluar un PLC.

Además de motivar y favorecer la comprensión, estas tareas funcionarán como los primeros elementos de recopilación de información sobre el avance en la adquisición de conceptos y contenidos por parte de los alumnos en el contexto de la evaluación formativa. En este sentido, las actividades propuestas son herramientas que resultan muy útiles para descubrir el grado de concienciación lingüística de nuestro alumnado.

La mayor parte del alumnado que ha cursado la asignatura está formado por docentes en activo y el fragmento restante está constituido por estudiantes que aspiran a convertirse en docentes. Por tal motivo, las actividades que hemos planteado proponen al estudiante realizar una investigación personal sobre diferentes temas, que relacionan la competencia en comunicación lingüística (CCL) y la concienciación lingüística con la didáctica de la lengua en Educación Infantil y Primaria en espacios de aplicación reales, como son, por ejemplo, el conocimiento de la legislación vigente en materia de educación en relación con las lenguas, la gestión de las lenguas en un centro escolar, la innovación en materia de enseñanza de lenguas y la concienciación lingüística en el contexto social inmediato del alumno de este máster universitario. 
El objetivo de la primera de las actividades (¿Qué lenguas se hablan en tu currículum?) consiste en conocer el currículum básico que afecta directamente el tratamiento de la o las lenguas oficiales de un mismo territorio en la realidad administrativa en la que vive el alumno. Se solicita que este demuestre conocer el currículum de Educación Infantil y Primaria para que, posteriormente, diseñe estrategias de enseñanza dentro de este contexto. Se pretende evaluar la comprensión del currículum, así como las formas de implementarlo en el aula. Este trabajo lo han realizado 39 alumnos.

La segunda actividad (Antropólogos lingüistas por un día) solicita al alumnado recoger en la realidad inmediata del alumno todo tipo de indicadores que le permitan conocer las lenguas — y sus variedades — a las que está expuesto un alumno de Educación Infantil y Primaria en el contexto geográfico en el que lleva a cabo (o le gustaría llevar a cabo) su actividad como docente. Ha sido implementada por 33 estudiantes.

La tercera actividad (Construyendo con mi PLC) supone idear un esbozo de un PLC aplicándolo a un centro escolar inventado, pero realista. En dicho esquema el alumno podrá, siguiendo lo aportado hasta ahora en esta asignatura, delimitar los agentes, procesos, objetivos y la temporalización para crear un armazón de esta herramienta. Ha sido realizada por 34 alumnos.

La cuarta actividad (Simulando imprevistos) consiste en examinar la puesta en funcionamiento y coordinación de un hipotético PLC, tomando como base el proyecto que han realizado en el tema "Recursos, herramientas y actividades para ejecutar el PLC”. El docente añadirá algún tipo de parámetro para simular y enseñar a anticiparse a posibles problemas que puede tener la implantación de un PLC en un centro. Llama la atención que solamente 16 alumnos ( 8 del grupo 1 y 8 del grupo 2 ) de un total de 45 han desarrollado esta tarea.

El objetivo de la quinta y última actividad (¿Funciona?) estriba en idear una serie de criterios de evaluación para nuestro ficticio PLC y comprobar si las prácticas que se llevarían a cabo serían las adecuadas para nuestro contexto. Se evaluará la comprensión de los conceptos tratados a lo largo de esta asignatura, así como la capacidad de observación, reflexión y análisis por parte del alumno para saber reconocer indicadores de evaluación que sirvan para mejorar la efectividad de un PLC. Este trabajo solamente fue realizado por 11 alumnos.

Si bien una de nuestras intenciones al plantear los temas y las actividades consistía en fomentar en los alumnos la concienciación lingüística a través de las dimensiones performativa, cognitiva, afectiva, social y de poder, tal efecto, y como medio de evalua- 
ción, hemos analizado las 39 actividades que corresponden al primero de los trabajos planteados a los alumnos con la idea de extraer marcadores que nos permitan constatar si hemos alcanzado nuestro objetivo.

Anteriormente se ha explicado que la primera actividad (¿Qué lenguas se hablan en tu currículum?) consiste en conocer el currículum básico que afecta directamente el tratamiento de la o las lenguas oficiales de un mismo territorio en la realidad administrativa. Dentro de esta tarea, al alumnado se le ha pedido que respondiera de una manera abierta, crítica y analítica a las siguientes cuestiones:

- ¿Cuántas lenguas contempla el currículum?

- ¿Sabes si existen más lenguas que se hablan en tu región y el currículum no las recoge?

- ¿Cuántas lenguas extranjeras se exige aprender a los alumnos? ¿Cuáles? ¿Por qué crees que es así? ¿Propondrías otra u otras?

En sus respuestas, de los 39 trabajos entregados, 17 alumnos han mostrado una alusión a la dimensión social. Como se ha expuesto aquí, esta dimensión hace referencia a la armonización social de contextos multiculturales y lingüísticamente diversos. Es interesante ver cómo seis de los alumnos españoles apelan a la enseñanza del árabe y ocho alumnos a la enseñanza del chino de una manera reglada, además de solicitar que esto se refleje en los currículum oficiales como un método para favorecer la intercomprensión en un contexto multicultural que predomina en la realidad de numerosas aulas españolas. Por su parte, 19 alumnos aluden a la dimensión afectiva. Recordemos que esta guarda relación con las actitudes, la motivación y la curiosidad acumuladas por medio de la conciencia lingüística. Resulta interesante apreciar cómo 11 alumnos colombianos aluden a la multiculturalidad y multilingüismo que ofrece este país americano, 65 lenguas indígenas y dos lenguas criollas según el Gobierno colombiano (Gobierno colombiano, s.f.). Es igualmente relevante apreciar cómo los alumnos españoles que residen en comunidades sin lengua cooficial hacen referencia a las variantes lingüísticas propias de su territorio, como sucede en el caso de la Fala en Extremadura, el dialecto riojano o la Fabla en Aragón. Finalmente 21 alumnos han aludido a la dimensión de poder. Esta está enfocada en el posible uso opresor que se hace de las lenguas por parte de distintas entidades. El inglés parece ser, según estos 21 estudiantes, una lengua que se habla por dos motivos principales: el primero tiene que ver con la existencia de una concepción de que el mundo es globalizado y, por tal motivo, el inglés actúa como un idioma universal para el plano tecnológico y digital; la segunda razón se relaciona con la necesidad de que los 
alumnos aprendan inglés para facilitar su inserción laboral. El aprendizaje del francés, del alemán o del chino mandarín se vincula con la misma motivación. Cabe añadir que otros alumnos (tanto españoles como colombianos) optaron por proponer el portugués como lengua extranjera debido a la cercanía de países lusoparlantes tanto en España como en Colombia. Así pues hemos podido constatar que un $43 \%$ del grupo de alumnos ha mostrado concienciación lingüística desde un punto de vista social; un $48 \%$ del grupo parece tener concienciación lingüística desde un punto de vista afectivo y finalmente un 52 \% del grupo alcanza la concienciación lingüística en su dimensión de poder.

Otra forma de analizar estos 39 trabajos es valiéndonos de la ayuda que nos ofrecen las tecnologías en relación con el procesamiento del lenguaje. En este sentido, una herramienta interesante que nos permite extraer del conjunto de actividades información útil sobre la acogida en el grupo de nuestra intención de concienciación lingüística es Linguakit, que permite diversos tipos de análisis. Para lo que aquí nos interesa nos ha resultado de interés hacer uso de las herramientas que ofrece el apartado de Análisis Lingüístico y, concretamente, la Frecuencia de palabras, una herramienta que permite analizar la diversidad de palabras de un texto según su frecuencia y tipo y, asimismo, también hemos empleado el Análisis Textual y, concretamente, el extractor multipalabra y la Frecuencia de adjetivos, que logra ampliar la búsqueda del extractor de palabras clave a términos y temas que necesitan más de una palabra.

\begin{tabular}{|c|c|}
\hline $\begin{array}{l}\text { Extractor } \\
\text { multipalabra }\end{array}$ & $\begin{array}{l}\text { Frecuencia de } \\
\text { adjetivos }\end{array}$ \\
\hline Lengua extranjera & extranjero \\
\hline $\begin{array}{l}\text { lineamientos } \\
\text { curriculares }\end{array}$ & básico \\
\hline currículo básico & lingüístico \\
\hline resultados pruebas & educativo \\
\hline lengua materna & primario \\
\hline lenguas extranjeras & español \\
\hline $\begin{array}{l}\text { competencia } \\
\text { comunicativa }\end{array}$ & oficial \\
\hline $\begin{array}{l}\text { habilidades } \\
\text { comunicativas }\end{array}$ & propio \\
\hline grupos étnicos & diferente \\
\hline
\end{tabular}




$\begin{array}{ll}\begin{array}{l}\text { lengua cooficial } \\ \text { lengua oficial }\end{array} & \begin{array}{l}\text { inglés } \\ \text { francés }\end{array} \\ \text { lengua vehicular } & \text { bilingüe } \\ \text { lengua castellana } & \text { cultural } \\ \text { uso oral } & \text { aragonés } \\ \text { intercambios } & \text { étnico } \\ \text { comunicativos } & \end{array}$

Este resultado léxico demuestra la concienciación lingüística de nuestros alumnos. Así, entre las multipalabras obtenidas observamos una alusión a la dimensión social, principalmente a través de la referencia a lenguas extranjeras, maternas, oficiales y cooficiales; también a la performativa y cognitiva a través de las alusiones a competencia comunicativa o habilidad comunicativa y, en cierto sentido, asistimos también a numerosas referencias a los textos legislativos que regulan el uso de las lenguas y, por tanto, a las instituciones de poder de las cuales emanan. Los resultados que nos ofrecen los adjetivos añaden interesantes matices. Se observa con claridad la dimensión social que tiene que ver con la convivencia armónica de diferentes lenguas: extranjero, español, oficial, inglés, francés, bilingüe, aragonés o étnico; pero quizás aquí pueda intuirse también una referencia a la dimensión afectiva mediante los términos diferente y propio.

Así pues, este breve análisis revela la necesidad de acercarse a un texto de diferentes maneras para poder extraer algunas conclusiones. Parece claro, no obstante, que puede demostrarse la adquisición de concienciación lingüística por parte de nuestros alumnos y esto se ha logrado por medio de la realización de esta actividad.

\section{Conclusiones}

El objetivo de este artículo consistía en demostrar cómo es posible trabajar la concienciación lingüística de los docentes o futuros docentes en formación de Educación Infantil y Primaria a través del PLC. En este sentido es importante considerar cómo estos docentes tendrán que enfrentarse en sus centros a realidades multiculturales y, a su vez, deberán enseñar a sus alumnos una serie de contenidos relacionados con la mencionada concienciación de acuerdo con lo que marca el currículum. Así pues hemos trabajado los conceptos de conciencia y concienciación lingüística, PLC y la presencia de algunas muestras de concienciación en los currículum de Educación Infantil y Primaria. 
Aludiendo al segundo objetivo presentado en este trabajo, el cual trata sobre los resultados de la propuesta didáctica llevada a cabo con los alumnos del Máster Universitario en Didáctica de la Lengua en Enseñanza Infantil y Primaria que han cursado la asignatura Diseño y Elaboración de un Plan Lingüístico de Centro, a este respecto se han planteado cinco actividades relacionadas con la creación de un PLC, a través de las cuales pretendíamos, asimismo, fomentar la concienciación lingüística de nuestros alumnos. Para demostrar que hemos alcanzado nuestro objetivo hemos tomado como muestra los textos de los 39 alumnos que han realizado la primera de las actividades y los hemos analizado tanto desde un punto de vista textual, valiéndonos de la extracción de fragmentos en los que hay muestra de alguna de las dimensiones relacionadas con la concienciación, y lingüístico, haciendo uso de una herramienta que permite extraer automáticamente tanto palabras aisladas, como multipalabras.

Los resultados de uno y otro análisis permiten concluir que los alumnos han alcanzado dicha reflexión lingüística propia de la concienciación a través de una actividad vinculada con el PLC y que, fundamentalmente, las dimensiones social, afectiva y de poder son las más relevantes en el contexto de los trabajos. Esto implica, por tanto, que el PLC supone una herramienta de extraordinaria importancia en la labor de concienciación lingüística.

\section{Referencias}

Association for Language Awareness -ALA-. (s.f.). About Association for Language Awareness. Retrieved from http://www.languageawareness.org/?page_id $=48 \mathrm{~b}$

Centro Virtual Cervantes. (s.f.). Conciencia lingüística. Recuperado de https://cvc.cervantes.es/ ensenanza/biblioteca_ele/diccio_ele/diccionario/conciencialinguistica.htm.

Consejo de Europa. (2002). Marco común europeo de referencia para las lenguas: aprendizaje, enseñanza, evaluación. Madrid: Ministerio de Educación, Cultura y Deporte, Secretaría General Técnica del MECD-Subdirección General de Información y Publicaciones, y Grupo Anaya.

Crystal, D. (2000). Diccionario de lingïística y fonética. Barcelona: Octaedro.

Garret, P. (2009). Language Education: Language Awareness. In J. Mey, Concise Encyclopedia of Pragmatics (pp. 435-438). 0xford: Elsevier.

Hawkins, E. (1984). Awareness of Language: An Introduction. Cambridge: Cambridge University Press.

James, C. \& Garret, P. (1991). Language Awareness in the Classroom. London: Longman. 
Jiménez, J. R. (2010). Evaluación de la competencia comunicativa. Intervención de la inspección. En I Congreso de Inspección de Andalucía: Competencias básicas y modelos de intervención en el aula. Recuperado de http://docplayer.es/9872138-Evaluacion-de-lacompetencia-comunicativa-intervencion-de-la-inspeccion-jose-ramon-jimenez-benitez. html

Kumaravadivelu, B. (1994). The Postmethod Condition: (E)merging Strategies for Second/ Foreign Language Teaching. Tesol Quarterly, 28(1), 27-48. Retrieved from https://cvc. cervantes.es/ensenanza/biblioteca_ele/antologia_didactica/enfoque_comunicativo/ kumaravadivelu06.htm

López, A. \& Trujillo, F. (Coords.). (2012). Guía para el diseño y puesta en marcha de un Proyecto Lingüístico de Centro. Madrid: Organismo Autónomo de Programas Educativos Europeos.

Lorenzo i Gales, N. (2015). Mejorar la comunicación para vivir y convivir en el mundo real. Cuadernos de Pedagogía, 458, 22-27.

Ministerio de Educación, Cultura y Deporte del gobierno de España. (2007). Real Decreto 1630/2006, de 29 de diciembre, por el que se establecen las enseñanzas mínimas del segundo ciclo de Educación Infantil. «BOE» núm. 4, de 4 de enero de 2007, páginas 474 a 482. Referencia: BOE-A-2007-185

Ministerio de Educación, Cultura y Deporte del gobierno de España. (2014). Real Decreto 126/2014, de 28 de febrero por el que se establece el currículo básico de la Educación Primaria. «BOE» núm. 52, de 1 de marzo de 2014. Referencia: BOE-A-2014-2222.

Ministerio de Educación, Cultura y Deporte del gobierno de España. (s.f.a). Elementos curriculares. Recuperado de http://www.mecd.gob.es/educacion-mecd/mc/lomce/elcurriculo/curriculoprimaria-eso-bachillerato/elementos.html

Ministerio de Educación, Cultura y Deporte del gobierno de España. (s.f.b). Educación Infantil. mecd.gob.es. Recuperado de http://www.mecd.gob.es/educacion-mecd/areaseducacion/ estudiantes/educacion-infantil.html

Onrubia, J. (2016). Aprender y enseñar en entornos virtuales: actividad conjunta, ayuda pedagógica y construcción del conocimiento. Revista de Educación a Distancia, 50, 1-14.

Real Academia Española. (s.f.). Diccionario. Recuperado de http://www.rae.es/

Sánchez, M. (2010). La Multi Language Learning Awareness y la importancia de su potenciación didáctica en clase de ELE. redELE Revista Electrónica de Didáctica Español Lengua Extranjera, (18), 90-100. Recuperado de https://sede.educacion.gob.es/publiventa/ 
redele-n-18-revista-electronica-de-didactica-espanol-como-lengua-extranjera/ ensenanza-lengua-espanola/20262

Trujillo, F. (2015). Un abordaje de la competencia lingüística. Cuadernos de Pedagogía, 458, 10-13. Recuperado de http://fernandotrujillo.es/un-abordaje-global-de-la-competenciaen-comunicacion-linguistica/

Trujillo, F. \& Rubio, R. (2014, mayo). El PLC como respuesta sistémica al reto de la competencia comunicativa en entornos educativos formales: propuesta de análisis de casos. Lenguaje y Textos, (39), 29-38.

Vez, J. M. (2004). Pacto curricular por una educación lingüística integral para una sociedad de la cultura. Revista de Estudios y Experiencias en Educación, 3 (5), 137-157. Recuperado de https://dialnet.unirioja.es/descarga/articulo/981992.pdf

Zapata, M. (2016). Secuenciación de contenidos y objetos de aprendizaje. Revista de Educación a Distancia, (n. ${ }^{0}$ extra 50), 1-29. 